\title{
Factorial Design Studies and Biopharmaceutical Evaluation of Simvastatin Loaded Solid Lipid Nanoparticles for Improving the Oral Bioavailability
}

\author{
Kovoru Krishnam Raju, ${ }^{1}$ Beeravelli Sudhakar, ${ }^{2}$ and Kolapalli Venkata Ramana Murthy ${ }^{2}$ \\ ${ }^{1}$ University College of Pharmaceutical Sciences, Acharya Nagarjuna University, Nagarjuna Nagar, Guntur, \\ Andhra Pradesh 522510, India \\ ${ }^{2}$ A.U. College of Pharmaceutical Sciences, Andhra University, Visakhapatnam, Andhra Pradesh 530003, India
}

Correspondence should be addressed to Kovoru Krishnam Raju; kkraju321@gmail.com

Received 12 November 2013; Accepted 16 December 2013; Published 13 February 2014

Academic Editors: C. Li and S. Santra

Copyright (C) 2014 Kovoru Krishnam Raju et al. This is an open access article distributed under the Creative Commons Attribution License, which permits unrestricted use, distribution, and reproduction in any medium, provided the original work is properly cited.

\begin{abstract}
Statins are HMG-CoA reductase inhibitors, which lower the cholesterol level through reversible and competitive inhibition; they are involved in the biosynthesis of cholesterol and other sterols. Simvastatin exhibits poor oral bioavailability $(<5 \%)$ and undergoes extensive microsomal metabolism by CYP enzymes. CYP3A4 is the major metabolizing enzyme that metabolizes lactone form of simvastatin and significantly lowers intestinal uptake. The hydrophobic properties of simvastatin prevent complete dissolution of the drug in the intestinal fluid which also contributes to its lower bioavailability. SLNs are alternative carrier system to polymeric nanoparticles. SLNs are in submicron size range $(1-1000 \mathrm{~nm})$. To overcome the hepatic first pass metabolism and to enhance the bioavailability, intestinal lymphatic transport of drugs can be exploited. In the present study, attempt has been made to prepare solid lipid nanoparticles of simvastatin to improve the bioavailability. SLNs of simvastatin were prepared with Trimyristin by hot homogenization followed by ultrasonication method. The SLNs were characterized for various physicochemical properties and analytical techniques like PXRD, DSC to study thermal nature and morphology of formulation and excipients. Promising results of the study indicated the applicability of simvastatin solid lipid nanoparticles as potential tools for improvement of bioavailability of poorly soluble drugs.
\end{abstract}

\section{Introduction}

Simvastatin is an antihyperlipidemic drug with plasma halflife of $3 \mathrm{hrs}$ and poor oral bioavailability $(<5 \%)$ due to the extensive first pass metabolism. Possible methods to avoid first pass metabolism include transdermal, buccal, rectal, and parenteral routes of administration. Oral route is the most commonly used and preferred route for the delivery of drugs, although several factors like $\mathrm{pH}$ of GIT, residence time, and solubility can affect drug absorption or availability by this route. Lymphatic delivery is an alternative choice to avoid first pass metabolism in oral drug delivery. Enhanced lymphatic transport of drugs avoids the hepatic first pass metabolism and improves bioavailability, because intestinal lymph vessels drain directly into thoracic duct and further into the venous blood, thus bypassing the portal circulation. The main function of the lymphatic system is to facilitate absorption of long chain fatty acids via chylomicron formation. Two different lipid-based approaches are known to enhance the lymphatic transport, which includes construction of a highly lipophilic prodrug and incorporation of drug in a lipid carrier. Lipidbased drug delivery systems have been reported to enhance the bioavailability of lipophilic drugs such as halofantrine and ontazolast by lymphatic transport of biosynthesized chylomicrons associated with the drugs $[1,2]$.

Solid lipid nanoparticles are alternative carrier system to polymeric nanoparticles. Solid lipid nanoparticles are in submicron size range $(1-1000 \mathrm{~nm})$. Solid lipid nanoparticles are physiologically acceptable, that is, method of SLN preparation avoids the usage of toxicologically less acceptable 
excipients. SLN can be administered through oral, parenteral, topical, and pulmonary routes. An additional advantage of SLN is lack of coalescences which increases physical stability. To overcome the hepatic first pass metabolism and to enhance the bioavailability, intestinal lymphatic transport of drugs can be exploited. Highly lipophilic compounds such as long chain triglycerides reach systemic circulation via the lymphatics. Majority of fatty acids with carbon chain length of 14 and above were found to be recovered in thoracic lymph. Size is one of the most important determinants of lymphatic uptake. Optimum size for lymphatic uptake is between 10 and $100 \mathrm{~nm}$ Swartz 2001 [3]. However, uptake is more selective and slower as particle size increases. Polymeric nano and microparticles uptake by lymphatic system through $\mathrm{M}$ cell of Peyer's patches of intestine was evidenced [4]. Nanoparticles coated with hydrophobic polymers tend to be easily captured by lymphatic cells in the body [5]. Lymphatic transport and bioavailability of halofantrine and ontazolast were enhanced after coadministration with lipidic vehicles [1, 2]. Recently Cavalli et al., 2000, reported that intraduodenally administered tobramycin loaded SLN showed sustained release and lymphatic targeting [6]. SLNs were found to improve the bioavailability of drug, for example, cyclosporin A and piribedil, Olbrich et al., 2002, and Demirel et al., 2001 [7, 8]. Zhang et al. 2010, reported that the oral bioavailability of SV after its incorporation into the lipid nanoparticles was improved by 3.37-fold for SLNs I and 2.55-fold for SLNs II compared with that from free SV in rats, and that of the SVA was significantly enhanced as well [9]. Comparative study of simvastatin loaded NLC verses SLN reported that NLC shows values 2.29 folds increased bioavailability compared to SLN [10].

\section{Materials and Methods}

Simvastatin was purchased from Dr. Reddy's Laboratories Limited, Hyderabad. Trimyristin (Dyanosan114) was obtained from Sasol, Germany. Soya lecithin was purchased from Sigma Aldrich, India. Poloxamer was purchased from Hi-Media, India. Acetonitrile and methanol were purchased from Rankem, India. Chloroform was purchased from Merck India.

\section{Solid Lipid Nanoparticles Prepared by Homogenization and Followed by Ultrasonication}

3.1. Method. Simvastatin loaded SLNs were prepared with triglyceride such as Trimyristin by hot homogenization followed by ultrasonication method. Simvastatin, triglyceride, and soya lecithin were heated above melting temperature of lipid and mixed rapidly with glass rod in hot melted condition. Poloxamer 188 dissolved in water and heated to equal temperature was added to the melted lipid phase and homogenization was carried out. Homogenization was carried out for three minutes in order to get coarse emulsion. Finally, the obtained preemulsion was subjected to ultrasonication for 20 minutes at temperature above the melting point
TABLE 1: Saturation solubility.

\begin{tabular}{lc}
\hline $\mathrm{pH}$ & $\mathrm{mg} / \mathrm{mL}$ \\
\hline 1.2 & $0.00212 \pm 0.27$ \\
4.5 & $0.000115 \pm 0.022$ \\
6.8 & $0.005932 \pm 0.6$ \\
8 & $0.000732 \pm 0.31$ \\
\hline
\end{tabular}

of the lipid. During formulation development process, the sonication time was studied at various time intervals like 5 , $10,15,20$, and 25 minutes, which was then finally optimized $[11,12]$.

3.2. Optimization of Formulation Variables (Surfactant and Coemulsifier). To study the effect of variables on characterization performance of SLNs, different batches were prepared using $3^{2}$ factorial designs. Amount of soya lecithin $\left(X_{1}\right)$ and Poloxamer $188\left(X_{2}\right)$ were selected as two independent variables which were varied at three levels, low level $(-1)$, medium level (0), and high level $(+1)$. Amounts of drug simvastatin (40 mg) and triglyceride (400 mg) concentrations were kept constant. Particle size $\left(Y_{1}\right)$ and zeta potential $\left(Y_{2}\right)$ were selected as dependent variables (Figures 1 and 2). Values of variables and batch codes are shown in Table 2. Design-Expert DX 8 software was used for the generation and evaluation of statistical experimental design [12].

3.3. Saturation Solubility. Excess quantity of simvastatin was added to various buffers, namely, $\mathrm{pH} 1.2$ (acid), $\mathrm{pH} 4.5$ (acetate), and phosphate buffers $\mathrm{pH} 6.8$ and $\mathrm{pH} 8$ and continuously shaken for 24 hours with the help of mechanical stirrer. One $\mathrm{mL}$ of sample was taken from each buffer and centrifuged for $15 \mathrm{~min}$ at $15,000 \mathrm{rpm} .20 \mu \mathrm{L}$ of the supernatant was injected to HPLC to determine the saturation solubility. Results are shown in Table 1.

3.4. Drug Content. Simvastatin SLN formulation was diluted 10 times with chloroform: methanol (1:1). Final dilution was made with acetonitrile and simvastatin content was determined by HPLC [9].

\section{Measurement of Particle Size, Polydispersity Index (PDI), and Zeta Potential (ZP) of SLN}

The particle size distribution, PDI, and ZP of simvastatin loaded SLNs were measured using a Malvern Zetasizer (Nano ZS90, UK). About $100 \mu \mathrm{L}$ of the prepared SLN dispersion was diluted to $5 \mathrm{~mL}$ with double distilled water and analyzed with Zetasizer [13].

4.1. Entrapment Efficiency. The percentage of drug entrapped in the lipid is determined by measuring the concentration of the drug in the aqueous phase by ultrafiltration method. The Centrisart devices are used in ultrafiltration method. Centrisant consist of filter membrane (Molecular wt cutoff 20,000 daltons) at the base of sample recovery chamber. 
TABLE 2: Characterization of solid lipid nanoparticles.

\begin{tabular}{lcccccc}
\hline Batch code & Poloxamer $(\mathrm{mg})$ & Soya lecithin $(\mathrm{mg})$ & Drug content \% & Size distribution $(\mathrm{nm})$ & Zeta potential (mV) & Polydispersibility \\
\hline F1 & 100 & 200 & $99.12 \pm 0.12$ & $125.6 \pm 1.25$ & $-45 \pm 4.14$ & $0.227 \pm 0.60$ \\
F2 & 100 & 150 & $96.02 \pm 0.11$ & $110.8 \pm 1.83$ & $-22 \pm 0.65$ & $0.175 \pm 0.22$ \\
F3 & 100 & 100 & $101.7 \pm 0.2$ & $157.8 \pm 0.07$ & $-25.1 \pm 1.2$ & $0.254 \pm 0.13$ \\
F4 & 150 & 200 & $99.2 \pm 0.13$ & $102.7 \pm 1.75$ & $-35.3 \pm 0.6$ & $0.287 \pm 0.24$ \\
F5 & 150 & 150 & $100 \pm 0.22$ & $101.7 \pm 1.30$ & $-31.3 \pm 0.8$ & $0.201 \pm 0.14$ \\
F6 & 150 & 100 & $98.22 \pm 0.05$ & $358.2 \pm 2.05$ & $-38 \pm 1.0$ & $0.445 \pm 0.52$ \\
F7 & 200 & 200 & $100.2 \pm 0.11$ & $99.7 \pm 0.9$ & $-31.6 \pm 1.2$ & $0.242 \pm 0.02$ \\
F8 & 200 & 150 & $99.13 \pm 0.02$ & $108.8 \pm 0.20$ & $-36.7 \pm 0.8$ & $0.21 \pm 0.22$ \\
F9 & 200 & 100 & $101.02 \pm 0.08$ & $104 \pm 0.08$ & $-37.7 \pm 1.8$ & $0.212 \pm 0.16$ \\
\hline
\end{tabular}

Note. ${ }^{*}$ Results are expressed as mean \pm standard deviation $(n=3)$.
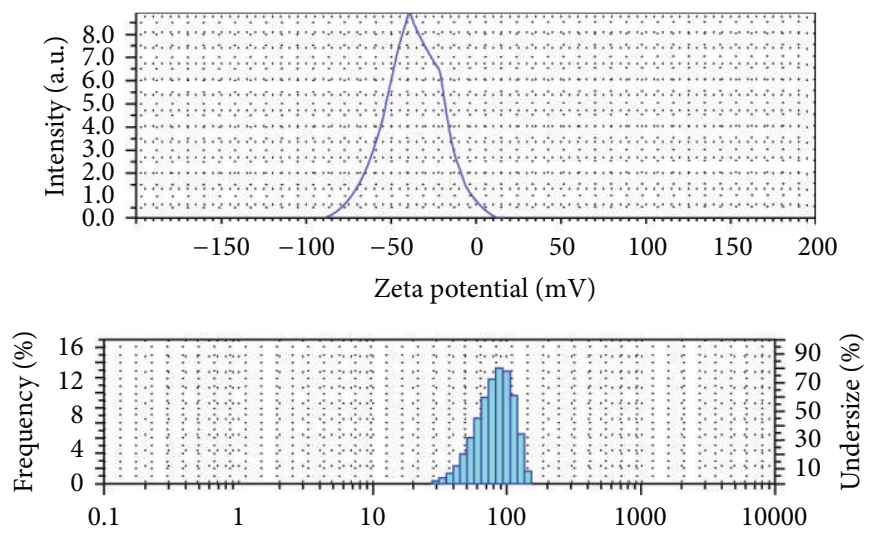

FIGURE 1: Zeta potential and particle size distribution of optimized SLN.

About $1 \mathrm{~mL}$ of undiluted sample is placed in the outer chamber on the top of the sample holder. The unit is centrifuged at 3,500 rpm for 15-20 min. The solid lipid nanoparticles along with the encapsulated drug remain in the outer chamber and the aqueous phase is moved into the sample recovery chamber through membrane. The amount of drug in the aqueous phase is estimated by HPLC $[13,14]$.

\section{Visualization by Transmission Electron Microscopy}

A drop of vesicle dispersion was applied on a carbon filmcovered copper grid. Excess dispersion was blotted from the grid with filter paper to form a thin-film specimen. The sample was then stained with $2 \%$ uranyl acetate, airdried, and examined under transmission electron microscope (Hitachi, H-7500) at a magnification of 50KX [13].

5.1. Lyophilization of the Optimized Formulation. Aqueous dispersion of SLN may not be stable physically for longer period of time, that is, drug release properties may be altered. To enable use of such formulation, it is necessary to convert such system into dry product by lyophilization. To prevent the particle aggregation, cryoprotectants were added before lyophilization. $5 \mathrm{~mL}$ of SLN formulation was taken along with $30 \%$ of trehalose solution $(5 \mathrm{~mL})$ in round bottom flask and carried out the freeze dried at $-45^{\circ} \mathrm{C}$. with the help of lyophilizer (Virtis Inc., New York) [14].

5.2. Differential Scanning Calorimeter. DSC analysis was carried out on Pyrus DSC Perklin Elmer. Heating rate of $10^{\circ} \mathrm{C} / \mathrm{min}$ was employed in the range of $20-240^{\circ} \mathrm{C}$. Analysis was performed under a nitrogen purge $(\mathrm{mL} / \mathrm{min})$. Standard aluminium crucibles $(40 \mu \mathrm{L})$ were used. About $10 \mathrm{mg}$ sample was taken for analysis and an empty pan is used as reference [14].

Preparation of mixtures of SLN component for DSC analysis:

(1) simvastatin,

(2) physical mixtures (PM) of simvastatin and triglyceride (Trimyristin),

(3) the physical mixture $(1: 10)$ of simvastatin and triglyceride was prepared. Physical mixture, tyophilized SLN, simvastatin and triglyceride was subjected the same thermal cycle as mentioned above.

5.3. Powder X-Ray Diffractometry (PXRD). Powder X-ray diffractometer (Siemens) was used for diffraction studies. PXRD studies were performed on the sample by exposing them to $\mathrm{CuK} \alpha$ radiation $(50 \mathrm{kv}, 34 \mathrm{~mA})$ and scanned from 3 to $45^{\circ}, 2 \theta$ at a scan step of $0.02^{0}$ and step time of $3^{0} / \mathrm{min}$. 

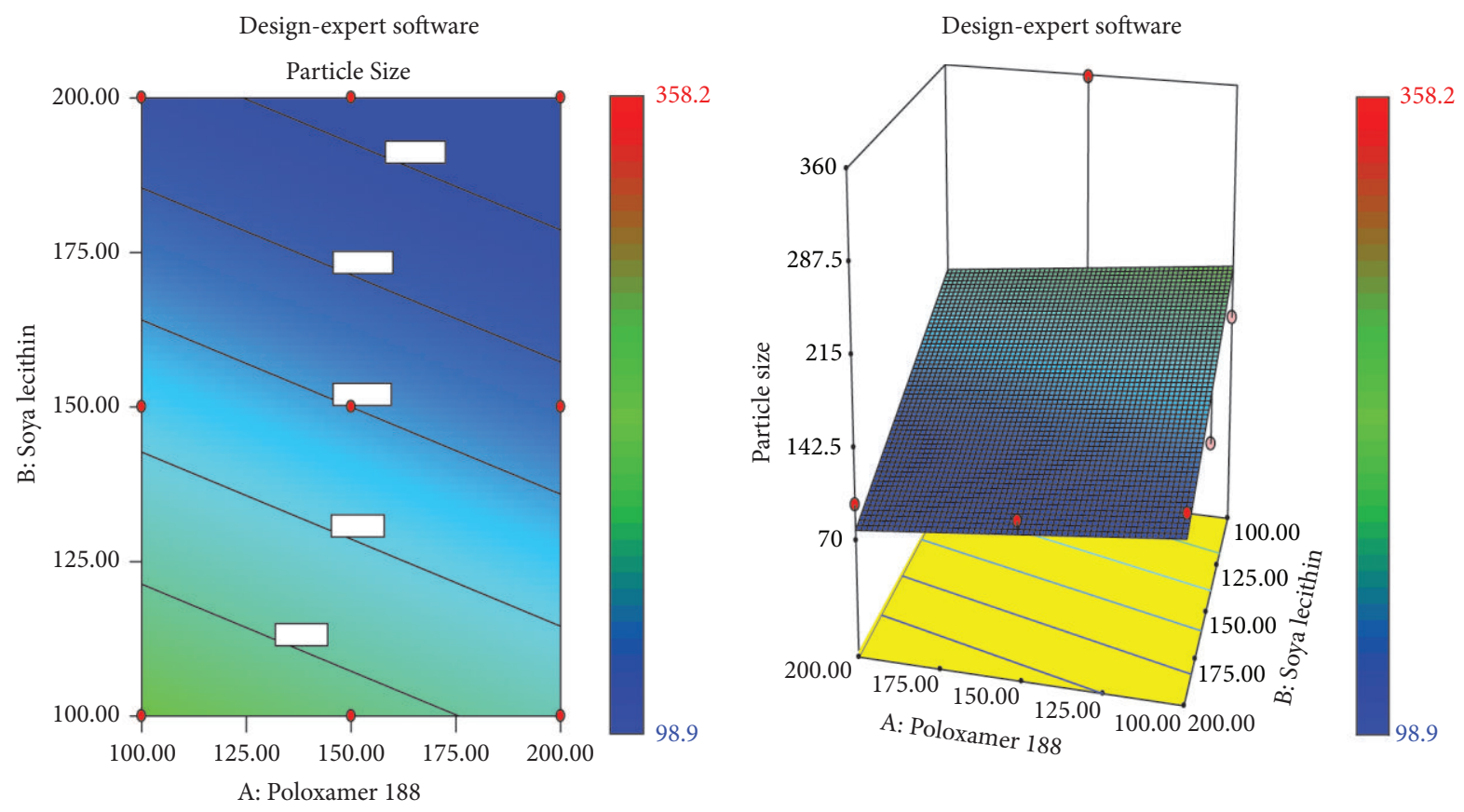
Particle size
- Design points

$$
\begin{aligned}
& X_{1}=\text { A: Poloxamer } 188 \\
& X_{2}=\text { B: Soya lecithin }
\end{aligned}
$$
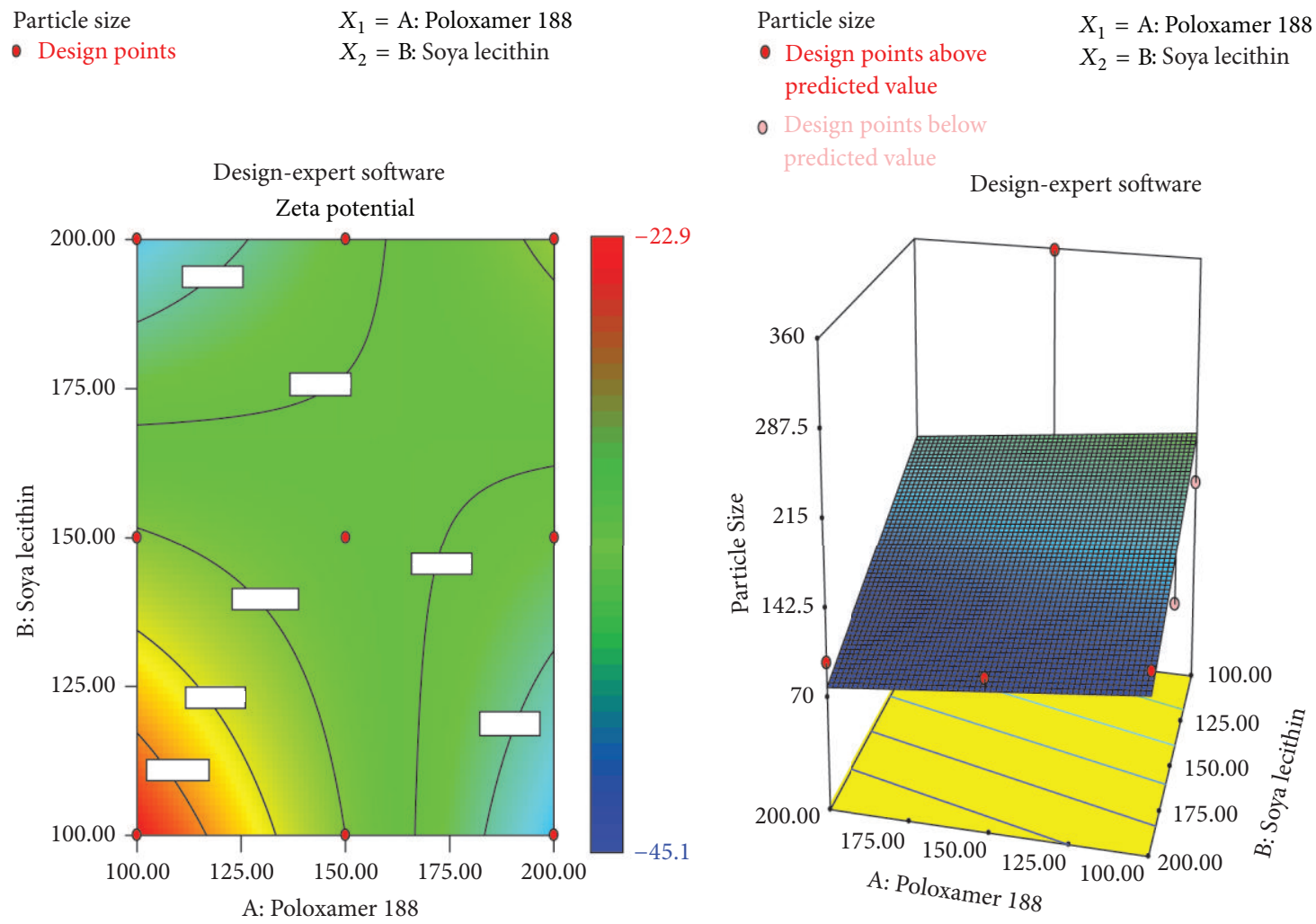

Design-expert software

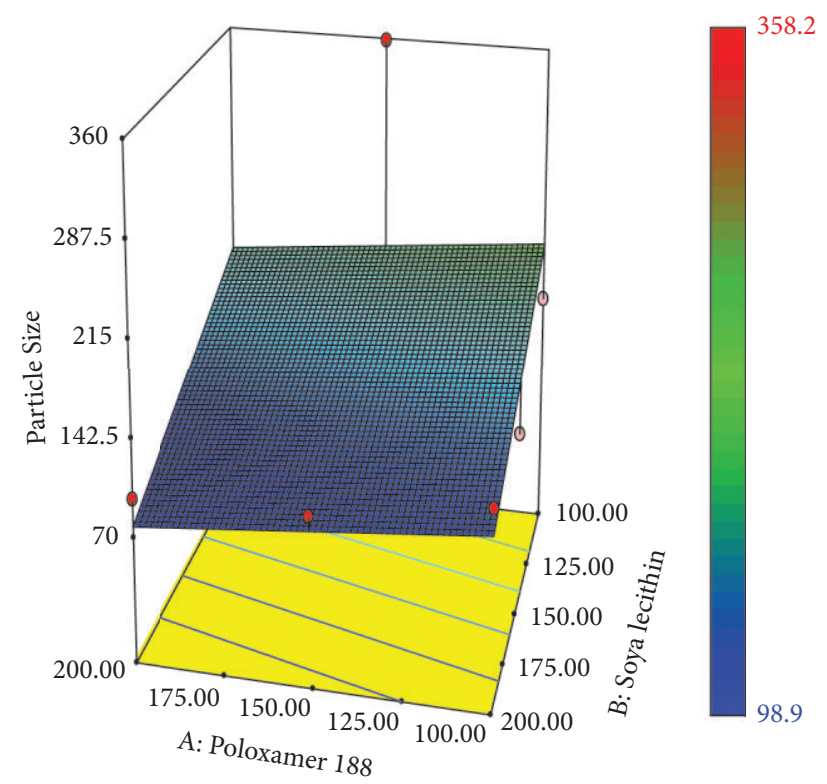

Zeta potential

- Design points

$$
\begin{aligned}
& X_{1}=\text { A: Poloxamer } 188 \\
& X_{2}=\text { B: Soya lecithin }
\end{aligned}
$$

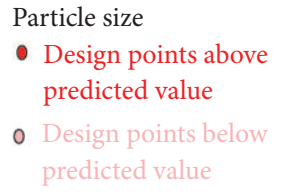

FIGURE 2: Surface response graphs and counter plots for particle size and zeta potential. 


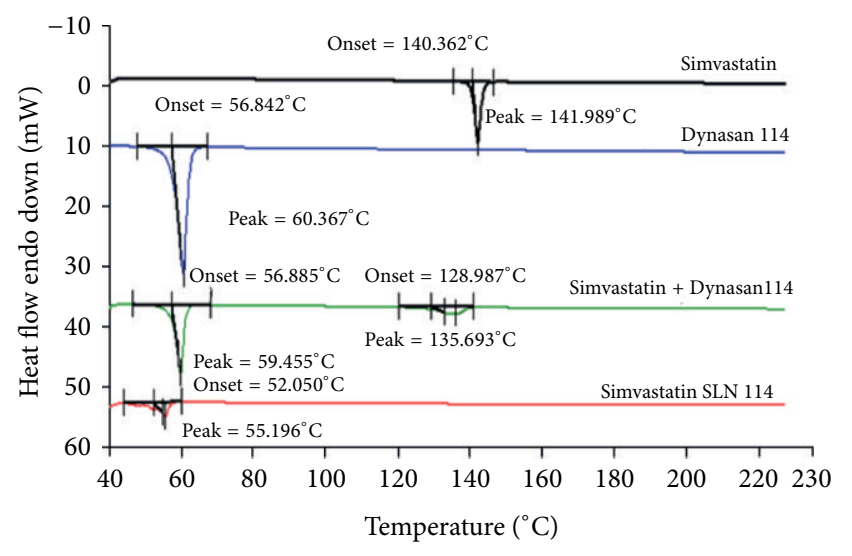

FIgURE 3: Overlaid thermograms of simvastatin loaded SLN.

Samples were used for PXRD analysis were the same as those of DSC analysis [15].

5.4. HPLC Specification. The chromatographic system consisted of Waters 2496 LC 10 AT solvent delivery pump equipped with a $20 \mu \mathrm{L}$ loop and Rheodyne sample injector and SPD 10 AVP Dual wavelength UV-visible detector. The column used for analysis was a C-18 Symmetry $(25 \mathrm{~cm} \times$ $4.6 \mathrm{~mm}$ ID). The mobile phase consisted of an aqueous buffer and an organic modifier (Buffer: Acetonitrile, $40: 60$ ). The aqueous buffer was sodium dihydrogen phosphate $(0.005 \mathrm{mM})$ and the phosphoric acid $(0.01 \mathrm{M})$ and the organic modifier was acetonitrile. The column temperature was maintained at $30^{\circ} \mathrm{C}$ and a flow rate of $1 \mathrm{~mL} / \mathrm{min}$ was used. Analysis was carried out using ultraviolet detector at wavelength of $238 \mathrm{~nm}$. Sensitivity selected was 0.001 a.u.f.s. The data was recorded and calculated using Winchrom software [16].

5.5. In Vitro Drug Release Studies by Franz Diffusion Cell. The in vitro drug release of simvastatin through dialysis membrane from SIMVA-SLN dispersion and dispersion of pure simvastatin was evaluated using modified Franz diffusion cell (Figure 6). Dialysis membrane having pore size $2.4 \mathrm{~nm}$ molecular weight cut-off 10,000 was used. Membrane was soaked in double distilled water for 12 hours before mounting in Franz diffusion cell. For comparative release studies simvastatin loaded SLN and pure simvastatin dispersion equivalent to $4 \mathrm{mg}$ was applied to the donor compartment. And the receptor compartment was filled with the dialysis media $(12 \mathrm{~mL}) 6.8 \mathrm{pH}$ phosphate buffer. At fixed time intervals of $0.5,1,2,4,6,8,12$, and $36 \mathrm{hrs}$, samples $(100 \mu \mathrm{L})$ were withdrawn from receiver compartment through side tube and replaced with suitable equivalent medium to maintain constant diffusion volume. Samples were analyzed by HPLC method to determine the simvastatin content [17].

\section{Results}

The saturation solubility studies indicated that $\mathrm{pH} 6.8$ phosphate buffer shows maximum solubility comparatively to other media. Average saturation solubility of simvastatin was

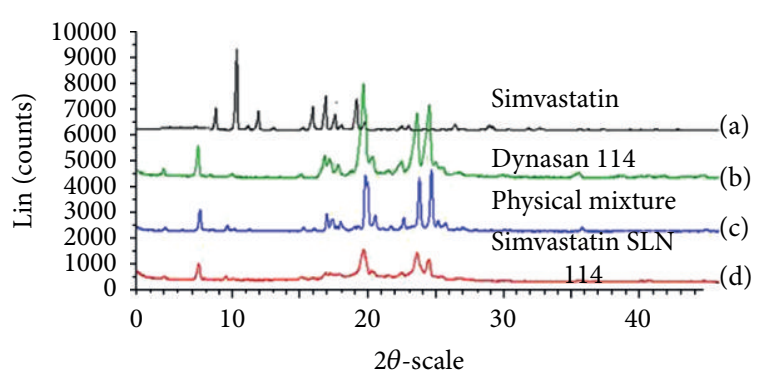
(a) Simvastatin
(c) Physical mixture
(b) Trimyristin
(d) Lyophilized SLN

FIGURE 4: Overlaid PXRD graph of simvastatin SLN.

TABLE 3: Effect of sonication time.

\begin{tabular}{lcc}
\hline Sonication time & Particle size $(\mathrm{nm})$ & Polydispersity index \\
\hline $5 \mathrm{~min}$ & $178.4 \pm 0.65$ & $0.381 \pm 0.02$ \\
$10 \mathrm{~min}$ & $133 \pm 1.6$ & $0.316 \pm 0.09$ \\
$15 \mathrm{~min}$ & $102 \pm 1.3$ & $0.296 \pm 0.03$ \\
$20 \mathrm{~min}$ & $99.7 \pm 0.9$ & $0.242 \pm 0.02$ \\
$25 \mathrm{~min}$ & $98 \pm 1.0$ & $0.222 \pm 0.31$ \\
\hline
\end{tabular}

Note. ${ }^{*}$ Results are expressed as mean \pm standard deviation $(n=3)$.

found to be $0.005932 \mathrm{mg} / \mathrm{mL}$. Hence, $\mathrm{pH} 6.8$ phosphate buffer was selected as vehicle to make SLN dispersions. Results are shown in Table 1.

The percentage drug content of the SLN formulations varied between $96.02 \pm 0.11 \%$ and $101.7 \pm 0.2 \%$. Hence, all the formulations were within the standard limits. This indicates uniform distribution of drug in each SLN formulation. The mean particle size was in the range of $99.7 \pm 0.9 \mathrm{~nm}$ to $358.2 \pm 2.05 \mathrm{~nm}$, and it was strongly affected by the selected variables. The polydispersity index (PDI) was in the range of $0.175 \pm 0.22$ to $0.445 \pm 0.52$ which indicated a narrow particle size distribution. Zeta potential values of prepared SLNs ranged from $-22.9 \pm 0.6$ to $-45.1 \pm 4.14 \mathrm{mV}$. For any liquid dosage form, surface charge is essential for its stability. SLNs were reported to have greater stability when compared to other colloidal dosage forms. Zeta potential value $> \pm 30 \mathrm{mV}$ is essential for effective stability and to inhibit aggregation. As the Poloxamer 188 concentration increased particle size was decreased and zeta potential of SLN decreased with increased concentration of Poloxamer 188 [18]. These results are shown in Table 2.

6.1. Effect of Sonication Time. Average particle size of SLN dispersions after 5, 10, 15, 20, and 25 minutes was shown in Table 3 . As the sonication time increased, average particle size was decreased up to 20 minutes and reduction of particle size was not observed after 20 minutes.

Depending on size, F7 batch was found to have optimum particle size distribution. Swartz, 2001 [3], reported that optimum particle size of SLN for lymph targeting was 10 $100 \mathrm{~nm}$. Hence, the F7 batch was optimized for further studies like entrapment efficiency and release studies. Entrapment efficiency of SLNs was determined after separating entrapped 
TABLE 4: Summary of model statistics for dependent variables.

\begin{tabular}{|c|c|c|c|c|c|c|c|c|c|c|c|}
\hline Model & $\begin{array}{l}\text { Sum of } \\
\text { squares }\end{array}$ & $\begin{array}{c}\text { Degrees of } \\
\text { freedom }\end{array}$ & Mean square & $R^{2}$ & $\begin{array}{c}\text { Adjusted } \\
R^{2}\end{array}$ & $\begin{array}{c}\text { Predicted } \\
R^{2}\end{array}$ & PRESS & s.d. & $F$ value & $\begin{array}{c}P \text { value } \\
\text { Prob }>F\end{array}$ & Remarks \\
\hline \multicolumn{12}{|c|}{ Response 1 (particle size $(\mathrm{nm}))$} \\
\hline Linear & 15423.02 & 2 & 7711.51 & 0.2764 & 0.0352 & -0.5608 & 87088.22 & 82.03 & 1.15 & 0.3789 & \multirow{3}{*}{ Suggested } \\
\hline Interactive & 183.60 & 1 & 183.60 & 0.2797 & -0.1525 & -1.6074 & 145485.1 & 89.66 & 0.02 & 0.8858 & \\
\hline Quadratic & 14921.87 & 2 & 7460.93 & 0.5471 & -0.2076 & -4.0030 & 279153.1 & 91.78 & 0.89 & 0.4985 & \\
\hline \multicolumn{12}{|c|}{ Response 2 (zeta potential $(\mathrm{mV})$ ) } \\
\hline Linear & 48.6416667 & 2 & 24.32083333 & 0.1291044 & - & -1.39076 & 900.7465 & 7.39505 & 0.44473 & 0.6605 & \multirow{3}{*}{ Suggested } \\
\hline Interactive & 170.3025 & 1 & 170.3025 & 0.5811203 & 0.329792 & -0.68016 & 633.0196 & 5.61815 & 5.395533 & 0.0678 & \\
\hline Quadratic & 59.0561111 & 2 & 29.52805556 & 0.7378666 & 0.300978 & -2.19533 & 1203.879 & 5.737652 & 0.896946 & 0.4951 & \\
\hline
\end{tabular}

TABLE 5: Melting peaks, enthalpies, and crystallinity of bulk lipid and lyophilized SLN.

\begin{tabular}{lccc}
\hline Parameter & Bulk & PM & Lyophilized SLN \\
\hline Melting peak $\left({ }^{\circ} \mathrm{C}\right)$ & 60.36 & 59.45 & 55 \\
Enthalpy $(\mathrm{j} / \mathrm{g})$ & 128.3 & 81.45 & 50.7 \\
Crystallinity $(\%)$ & 100 & & \\
\hline
\end{tabular}

and unentrapped drug by ultrafiltration. The F7 batch was found to have $95.20 \pm 1.02 \%$ entrapment efficiency, due to its carbon long chain and phase transition temperature.

Table 4 suggests the suitable model for further analysis. As per the $P$ values of particle size, linear model is best fit for the given data because it has the $P$ value less than interactive and quadratic models. Further, the $R$-square value of this linear equation is 0.2764 which indicates that $27.64 \%$ of variation is obtained for the dependent variable due to the independent variable. For the zeta potential, the interactive procedure is best suited when compared with linear and quadratic models since the $P$ value of the interactive model is smaller than the remaining two models. The $R^{2}$ value of 0.5811 explains that the variation obtained due to this independent variable is $58.115 \%$.

Regression equations:

$$
\begin{aligned}
& \text { Particle Size }=+140.94-13.75 * A-48.80 * B \\
& \begin{aligned}
\text { Zeta Potential }= & -33.74-2.15 * A-1.87 * B \\
& +6.53 * A * B .
\end{aligned}
\end{aligned}
$$

\section{DSC Studies}

The thermograms of lyophilized simvastatin SLN of Trimyristin did not show the sharp melting peak for the simvastatin around $240^{\circ} \mathrm{C}$ (Figure 3 ). This shows that simvastatin was not in crystalline state. However, this was confirmed by absence of melting peak of simvastatin in the physical mixture $(1: 10)$. This suggests that simvastatin was not in crystalline state but it was in amorphous state. Similar results were reported by [19] stating that rapid quenching of the microemulsion does not allow the drug to crystallize. [20], put forward the DSC analysis of the Camptothecin SLN preparation prepared by high pressure homogenization, which showed that Camptothecin was in amorphous state. In our method, lipids and simvastatin were melted and mixed thoroughly above the melting temperature of lipid and subsequent addition of hot surfactant phase allows homogenous dispersion of drug in lipid. Furthermore, method of preparation (homogenization followed by sonication) and presence of surfactant could not allow the drug to crystallize. Degree of crystallinity of lyophilized SLN was calculated by comparing the enthalpy of SLN with enthalpy of bulk lipid according to [21] (Table 5). Enthalpy of bulk lipid is being taken as $100 \%$. Enthalpy of SLN was calculated on the basis of total weight taken. Melting points of Trimyristin in SLN form were depressed when compared to melting points of corresponding bulk triglyceride. These suggest that triglyceride in SLNs might be in $\beta 1$ form $[18,22]$. Freitas and Müller, 1998, reported that Compritol in SLN was $\beta 1$ or $\beta 1$ form. This melting point depression might be due to small particle size (nanometer range), their high specific surface area, and the presence of surfactant. This was confirmed by physical mixture where such type of depression was not found. This melting point effect can be attributed to the Kelvin effect.

7.1. Powder X-Ray Diffractometry (PXRD). PXRD patterns of simvastatin exhibit sharp peaks at $2 \theta$ scattered angles indicating crystalline nature of simvastatin. Results were shown in Figure 4. Reduced intensity was observed for the characteristic peaks in the simvastatin lyophilized SLN of Trimyristin, when compared with pure simvastatin and physical mixture. The reduced intensity suggests greater decrease in crystallinity of simvastatin in the prepared lyophilized SLN.

\section{In Vitro Drug Release Studies}

Depending on particle size distribution, zeta potential, and entrapment efficiency SLN-7 batch formulation was optimized for drug release studies and compared with pure simvastatin dispersion. The cumulative \% release of simvastatin in 36 hrs from SIMVA-SLN dispersion was significantly retarded $(37.08 \pm 0.22 \%)$ compared to that from dispersion of pure drug $(98.08 \pm 1.2 \%)$. Thus, very slow release was observed. These suggest that simvastatin was homogenously dispersed in the lipid matrix. The drug release kinetics 


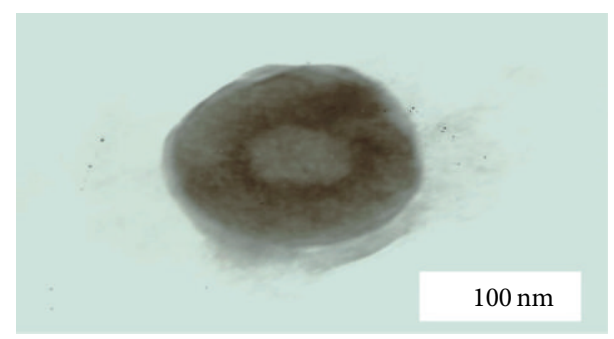

FIGURE 5: Surface morphology of SLN by TEM studies.

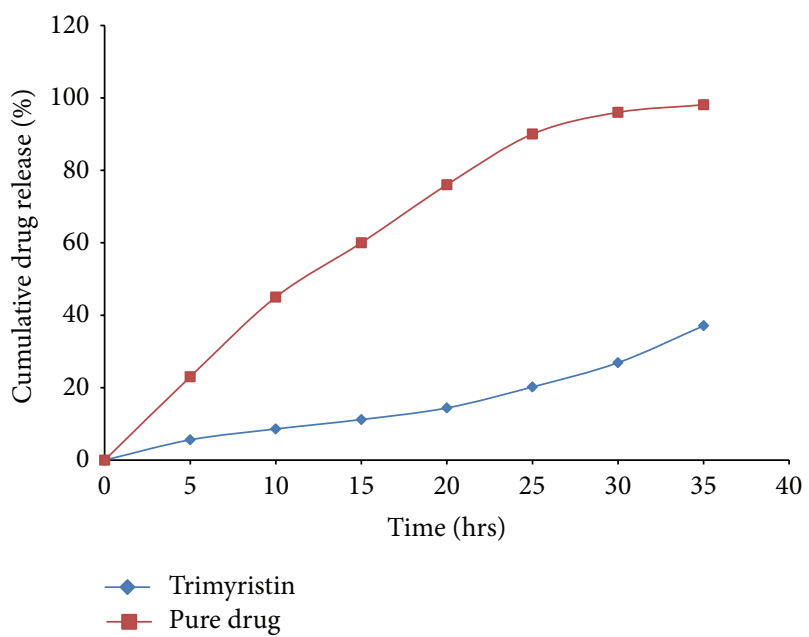

FIGURE 6: Comparative In-vitro drug release studies.

indicate that drug release followed zero order and nonFickian diffusion manner and absence of any burst release, indicates complete entrapment of drug in the lipid matrix without any adsorption of simvastatin on the surface of SLNs (Figure 5).

\section{Conclusion}

Simvastatin loaded solid lipid nanoparticles were papered by using $3^{2}$ factorial design to yield an optimized formulation (F7) having lowest particle size and higher entrapment efficiency that controlled drug release for $36 \mathrm{hrs}$. Promising results of the study indicated the applicability of simvastatin solid lipid nanoparticles as potential tools to improve the bioavailability of poorly soluble drugs.

\section{Conflict of Interests}

The authors declare that there is no conflict of interests regarding the publication of this paper.

\section{References}

[1] R. Holm, H. Tønsberg, E. B. Jørgensen, P. Abedinpour, S. Farsad, and A. Müllertz, "Influence of bile on the absorption of halofantrine from lipid-based formulations," European Journal of Pharmaceutics and Biopharmaceutics, vol. 81, no. 2, pp. 281287, 2012.

[2] D. J. Hauss, S. E. Fogal, J. V. Ficorilli et al., "Lipid-based delivery systems for improving the bioavailability and lymphatic transport of a poorly water-soluble LTB4 inhibitor," Journal of Pharmaceutical Sciences, vol. 87, no. 2, pp. 164-169, 1998.

[3] M. A. Swartz, "The physiology of the lymphatic system," Advanced Drug Delivery Reviews, vol. 50, no. 1-2, pp. 3-20, 2001.

[4] F. Delie, "Evaluation of nano- and microparticle uptake by the gastrointestinal tract," Advanced Drug Delivery Reviews, vol. 34, no. 2-3, pp. 221-233, 1998.

[5] Y. Nishioka and H. Yoshino, "Lymphatic targeting with nanoparticulate system," Advanced Drug Delivery Reviews, vol. 47, no. 1, pp. 55-64, 2001.

[6] R. Cavalli, M. R. Gasco, P. Chetoni, S. Burgalassi, and M. F. Saettone, "Solid lipid nanoparticles (SLN) as ocular delivery system for tobramycin," International Journal of Pharmaceutics, vol. 238, no. 1-2, pp. 241-245, 2002.

[7] C. Olbrich, O. Kayser, and R. H. Müller, "Lipase degradation of Dynasan 114 and 116 solid lipid nanoparticles (SLN) — effect of surfactants, storage time and crystallinity," International Journal of Pharmaceutics, vol. 237, no. 1-2, pp. 119-128, 2002.

[8] M. Demirel, Y. Yazan, R. H. Müller, F. Kiliç, and B. Bozan, "Formulation and in vitro-in vivo evaluation of piribedil solid lipid micro- and nanoparticles," Journal of Microencapsulation, vol. 18, no. 3, pp. 359-371, 2001.

[9] Z. Zhang, H. Bu, Z. Gao, Y. Huang, F. Gao, and Y. Li, "The characteristics and mechanism of simvastatin loaded lipid nanoparticles to increase oral bioavailability in rats," International Journal of Pharmaceutics, vol. 394, no. 1-2, pp. 147-153, 2010.

[10] R. Tiwari and K. Patha, "Nanostructured lipid carrier versus solid lipid nanoparticles of simvastatin:Comparative analysis lysof characteristics, pharmacokinetics and tissue uptake," International Journal of Pharmaceutics, vol. 415, pp. 232-243, 2011.

[11] A. Narala and K. Veerabrahma, "Preparation, Characterization and Evaluation of Quetiapine Fumarate Solid Lipid Nanoparticles to Improve the Oral Volume," Bioavailability Journal of Pharmaceutics, vol. 2013, Article ID 265741, 7 pages, 2013.

[12] M. Shah and K. Pathak, "Development and statistical optimization of solid lipid nanoparticles of simvastatin by using 23 fullfactorial design," AAPS PharmSciTech, vol. 11, no. 2, pp. 489496, 2010.

[13] J. M. Aceves-Hernández, J. Hinojosa-Torres, I. NicolásVázquez, R. M. Ruvalcaba, and R. M. L. García, "Solubility of simvastatin: a theoretical and experimental study," Journal of Molecular Structure, vol. 995, no. 1-3, pp. 41-50, 2011.

[14] J. J. Bojjola and L. D. Russell, Electron Microscopy Principles and Techniques for Biologists, Janes and Bartlett publishers, Sudbury, Mass, USA, 2nd edition, 1998.

[15] G. Suresh, K. Manjunath, V. Venkateswarlu, and V. Satyanarayana, "Preparation, characterization, and in vitro and in vivo evaluation of lovastatin solid lipid nanoparticles," AAPS PharmSciTech, vol. 8, no. 1, pp. E162-E170, 2007.

[16] V. K. Venishetty, R. Chede, R. Komuravelli, L. Adepu, R. Sistla, and P. V. Diwan, "Design and evaluation of polymer coated carvedilol loaded solid lipid nanoparticles to improve the oral bioavailability: a novel strategy to avoid intraduodenal administration," Colloids and Surfaces B, vol. 95, pp. 1-9, 2012.

[17] F. Dias Marques-Marinho, B. Diniz Freitas, J. Carlos da Costa Zanon, I. Afonso Reis, A. Alves Lima, and C. Duarte ViannaSoares, "Development and validation of a RP-HPLC method for 
simvastatin capsules," Current Pharma Analysis, vol. 9, no. 1, pp. $2-12,2013$.

[18] C. Freitas and R. H. Müller, "Effect of light and temperature on zeta potential and physical stability in solid lipid nanoparticle (SLN) dispersions," International Journal of Pharmaceutics, vol. 168, no. 2, pp. 221-229, 1998.

[19] M. Gambhire, M. Bhalekar, and B. Shrivastava, "Bioavailability assessment of simvastatin loaded solid lipid nanoparticles after oral administration," Asian Journal of Pharmaceutical Sciences, vol. 6, no. 6, pp. 251-258, 2011.

[20] C. Bocca, O. Caputo, R. Cavalli, L. Gabriel, A. Miglietta, and M. R. Gasco, "Phagocytic uptake of fluorescent stealth and non-stealth solid lipid nanoparticles," International Journal of Pharmaceutics, vol. 175, no. 2, pp. 185-193, 1998.

[21] S. Martins, I. Thob, I. Reimoldc et al., "Brain delivery of camptothecin by means of solid lipid nanoparticles: formulation design, in vitro and in vivo studies," International Journal of Pharmaceutics, vol. 439, no. 1-2, pp. 49-62, 2012.

[22] C. Freitas and R. H. Müller, "Spray-drying of solid lipid nanoparticles (SLNTM)," European Journal of Pharmaceutics and Biopharmaceutics, vol. 46, pp. 145-151, 1998. 

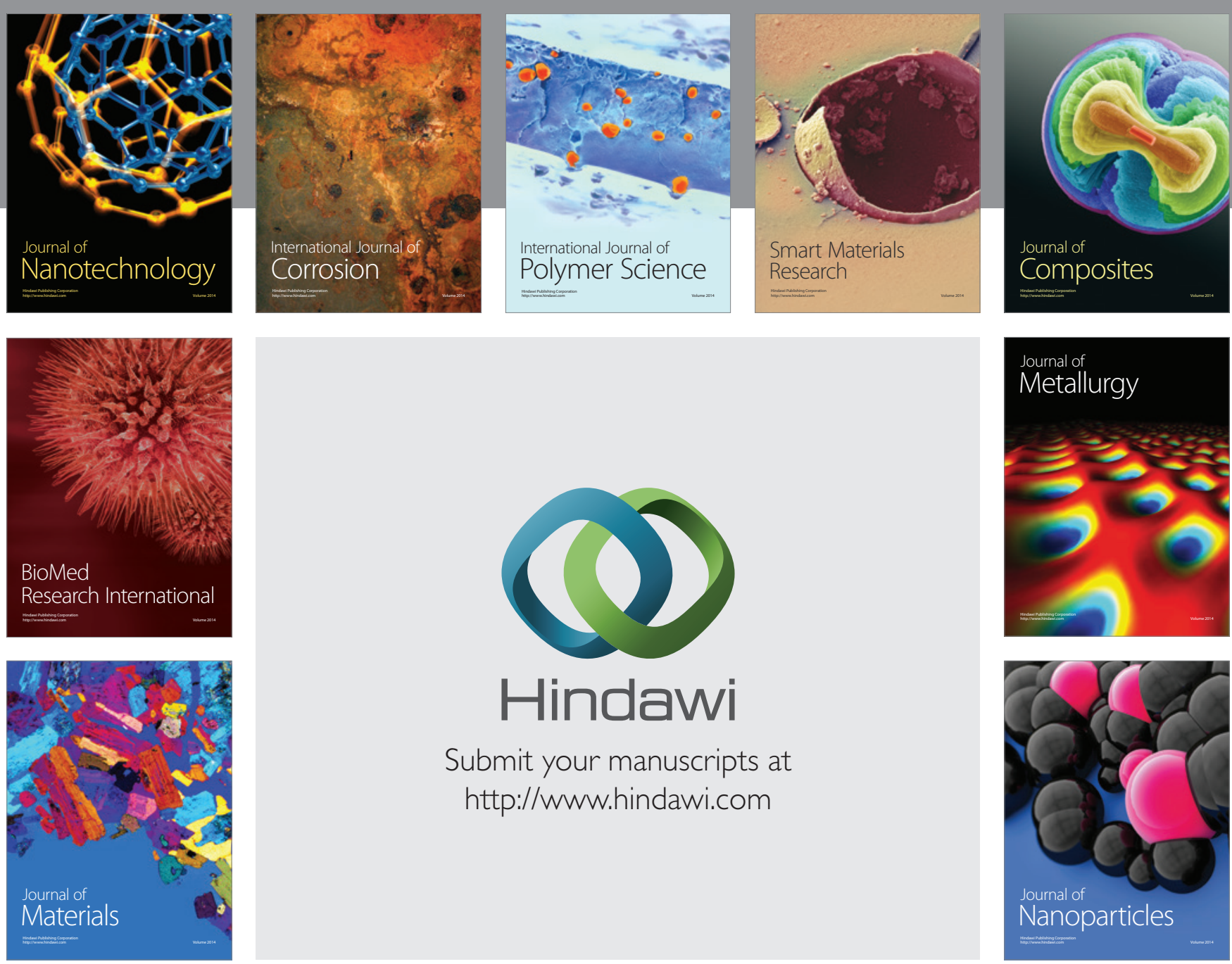

Submit your manuscripts at http://www.hindawi.com
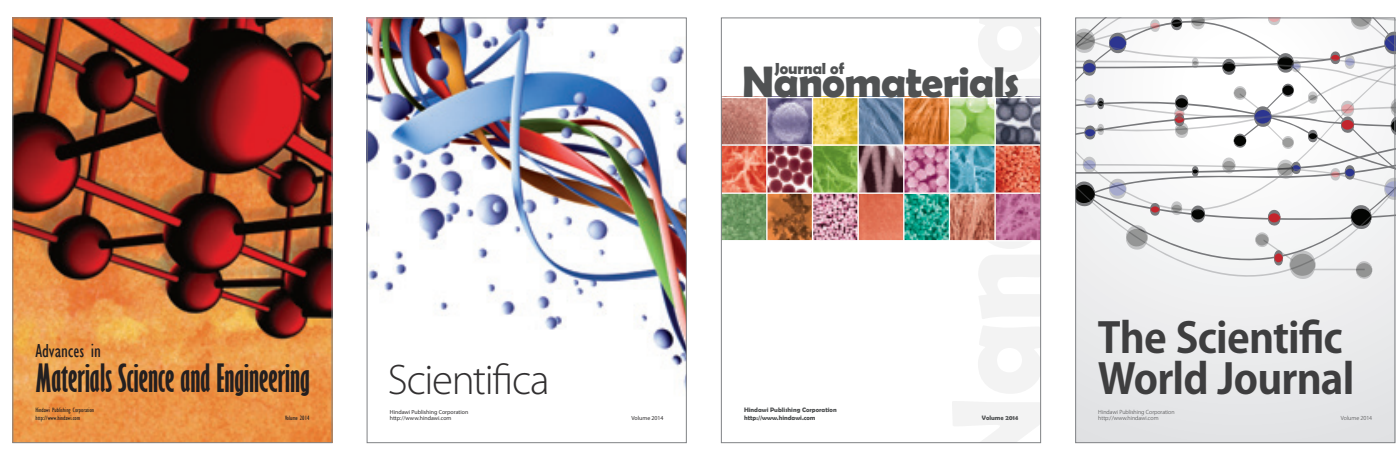

\section{The Scientific World Journal}
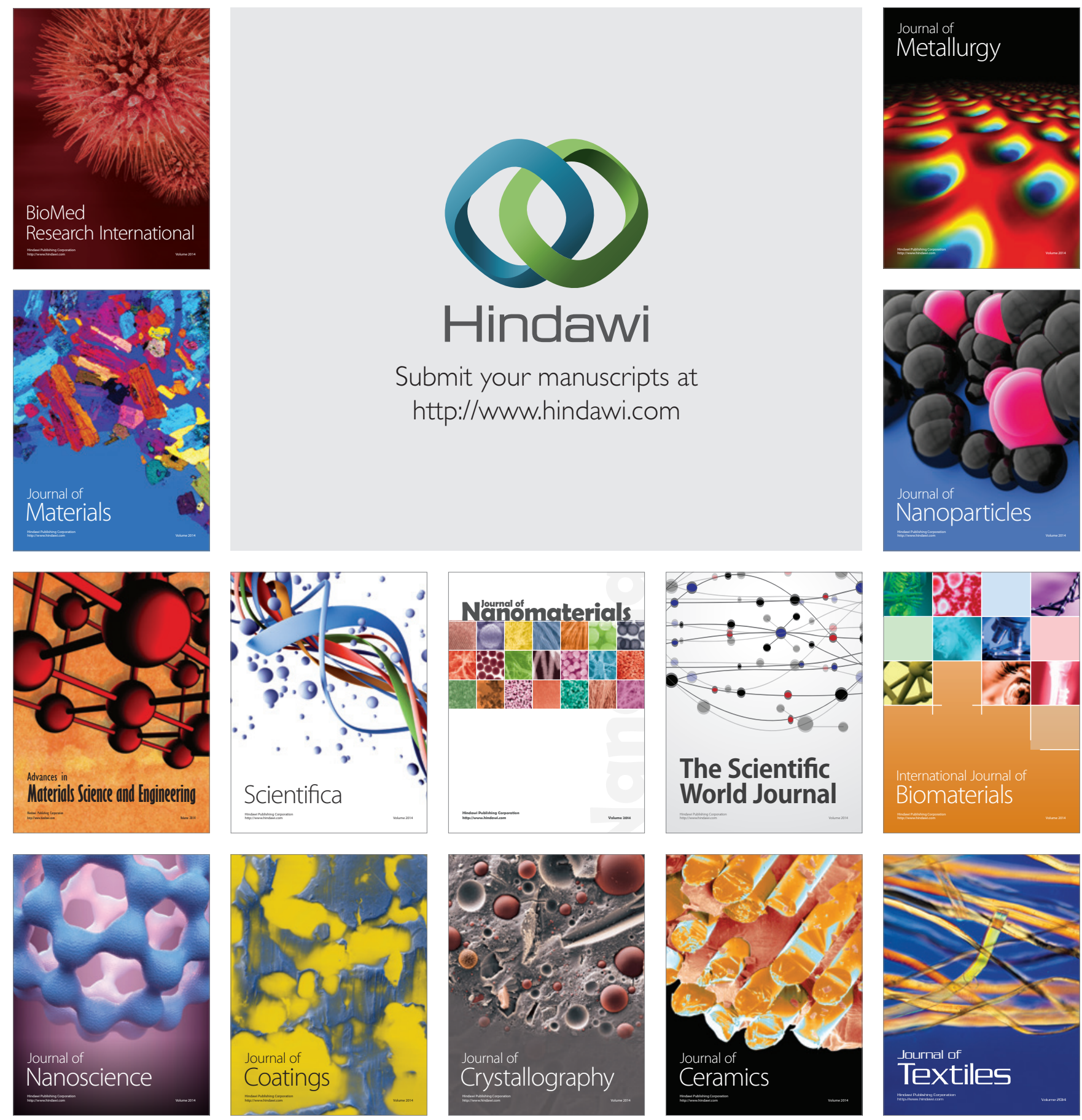